1. P. ApPELl, "Sur une classe de polynômes à deux variables et le calcul approché des intégrales doubles," Annales de la Faculté des Sciences de Toulouse, v. 4, 1890, p. H.1-H.20.

2. C. Birindelli, "Su nuove formule interpolatorie del Picone per funzioni in piu variabili e loro contributo al calcolo numerico degli integrali multipli," Compositio Mathematica, v. 10, 1952, p. 117-167.

3. W. BURnside, "An approximate quadrature formula," Messenger of Math., v. 37, 1908, p. $166-167$.

4. C. F. GAuss, "Methodus nova integralium valores per approximationem inveniendi," Werke, Gö̀ttingen, v. 3, 1866, p. 163-196.

5. P. C. Hammer, O. J. Marlowe, \& A. H. Stroud, "Numerical integration over simplexes and cones," $M T A C$, v. 10, 1956, p. 130-137.

6. P. C. Hammer \& Arthur H. Stroud, "Numerical integration over simplexes," $M T A C$, v. 10, 1956, p. 137-139.

7. Preston C. Hammer \& A. Wayne Wymore, "Numerical evaluation of multiple integrals I," $M T A C$, v. 11,1957, p. 59-67.

8. J. C. Maxwell, "On approximate multiple integration between limits by summation," Camb. Phil. Soc., Proc., v. 3, 1877, p. 39-47.

9. M. Picone, "Vedute generali sull' interpolazione e qualche loro conseguenza," Annali della Scuola Normale Superiore di Pisa, Ser. III, v. 5, 1951, p. 193-244.

10. Michael Sadowsky, "A formula for approximate computation of a triple integral," Amer. Math. Mon., v. 47, 1940, p. 539-543.

11. G. Szegö, Orthogonal Polynomials, Amer. Math. Soc. Colloquium Publication, v. 23, New York, 1939.

12. G. W. TyLER, "Numerical integration of functions of several variables," Can. J. Math., v. 5,1953 , p. $393-412$.

13. Richard von Mises, "Numerische Berechnung mehrdimensionaler Integrale," $Z$. Angew. Math. Mech., v. 34, 1954, p. 201-210.

\title{
On a Generalization of the Prime Pair Problem
}

1. Introduction. One of the many unsolved problems in the theory of prime numbers concerns the celebrated conjecture by A. de Polignac [1] about primes which differ by two. Prime pairs (or twin primes) are generally believed to constitute an infinite set but to date no one has succeeded in proving or disproving this conjecture.

It was established by Brun [2] that the sum of the reciprocals of all prime pairs is bounded but whether this boundedness is due to the finiteness of the set or to the "thinness" of an infinite set has not been determined.

2. Prime n-tuples. In generalizing from prime pairs to prime $n$-tuples we must first define what is meant by a prime $n$-tuple.

Definition: a prime $n$-tuple is a set of $n$ odd primes $p_{1}, p_{2}, \cdots p_{n}$ such that the difference $\Delta n=p_{n}-p_{1}$ is a non-trivial minimum.

The meaning of "non-trivial" will now be explained. For $n=3$ we can exhibit a set of three consecutive odd primes such that $\Delta 3=4$ (i.e., $3,5,7$ ). We consider this a trivial minimum, however, because (with one exception) in every set of three consecutive odd integers one of the integers in the set is composite since it is divisible by 3 . The single exception to this rule is the case where the number divisible by 3 is the number 3 itself. Generally, we consider a set of $n$ consecutive primes a trivial prime $n$-tuple if one of the primes of the set is always a factor of one of the terms of any sequence of consecutive odd integers having the same value for $\Delta n$. 
3. The generalized problem. We are now in a position to state the generalized prime pair problem:

(1) For a given $n$, what is the value of $\Delta n$ ?

(2) For a given $n$, is the set of primes which yield $\Delta n$ finite or infinite?

Clearly, the second question remains unanswered for all $n \geq 2$. The first question, however, can be answered for specific values of $n$ by establishing the following conditions:

(A) Show that for every set of $n$ odd primes $p_{1}, p_{2}, \cdots, p_{n}$ that $\Delta n>U$ where $U$ is some positive integer.

(B) Exhibit a set of $n$ odd primes such that $\Delta n=U+2$.

4. An example. The procedure followed in establishing condition (A) will be illustrated for $n=8$. Beginning with 1 , write down 14 consecutive odd numbers $(\bmod 10)$

\section{$\begin{array}{lllllllllllllll}13 & 5 & 7 & 1 & 3 & 5 & 7 & 9 & 3 & 5 & 7 .\end{array}$}

Now, assuming the first number represents a prime, the 2nd or 3rd and every 3 rd number thereafter is divisible by 3 ; one of the 2 nd, 3 rd, 4 th, or 5 th and every 5 th number thereafter is divisible by 5 , etc., for $7,11,13, \ldots$ Taking every possible combination of assigning factors there will be one or more combinations which leave a maximum number of terms of the sequence which have not been assigned factors. Placing the factors which have been assigned underneath the terms to which they have been assigned, the maximum combination for this sequence is

$$
\begin{array}{rrrrrrrrrrrrr}
13 & 5 & 7 & 9 & 1 & 3 & 5 & 7 & 9 & 1 & 3 & 5 & 7 \\
& 3 & & & 3 & & 5 & 3 & & & 3 & 5 \\
& 5 & & & 7 & & & & & & & 7
\end{array}
$$

We need not assign any further factors since $11,13,17, \cdots$, can always be assigned in such a fashion as to involve only terms which have already been assigned factors. If the same procedure is followed for sequences of 14 odd numbers beginning with 3,7 , and 9 , respectively, it is found that there is no combination that will leave more than 8 unassigned terms and two combinations that leave 8 unassigned terms, i.e.,

$\begin{array}{rrrrrrrrrrrrrr}3 & 5 & 7 & 9 & 1 & 3 & 5 & 7 & 9 & 1 & 3 & 5 & 7 & 9 \\ 5 & 3 & & & 3 & 5 & & 3 & & & 3 & & \\ 7 & & & & & & & 7 & & & 5 & & \end{array}$

and

$$
\begin{array}{llllllllllllll}
7 & 9 & 1 & 3 & 5 & 7 & 9 & 1 & 3 & 5 & 7 & 9 & 1 & 3 \\
& & 3 & & 5 & 3 & & & 3 & 5 & & 3 & &
\end{array}
$$

If we repeat the same process for 13 consecutive odd numbers we find there is no way to assign the factors to leave 8 terms unassigned. Since the difference between the first and 13th odd number is 24 we clearly have condition (A) satisfied when $U=24$. Now, if we can find a set of 8 consecutive odd primes $(\bmod 10)$ equal, respectively, to the 8 unassigned terms in any one of the three sequences 
determined above we shall have proved that $\Delta 8=26$. The following primes complete the proof.

$$
11,13,17,19,23,29,31,37 .
$$

When conditions (A) and (B) have been established, the procedure described here gives all the essentially different poissible types of $n$-tuples for a given $n$ and by solving one or more linear indeterminate equations the exact form of each type of $n$-tuple can be obtained. Thus, for $n=8$, there are, at most, three different types of 8-tuples, viz.,
(a) $210 x+11,13,17,19,23,29,31,37$
(b) $210 x+173,179,181,187,191,193,197,199$
(c) $30 x+17,19,23,29,31,37,41,43$.

5. Some further questions. The preceding discussion raises some new questions:

(3) Are there values of $n$ such that $\Delta n>U+2$ ?

The writer has verified that $\Delta n=U+2$ for all $n \leq 10$. On the other hand an exhaustive search of the first two billion numbers failed to disclose a single 19-tuple of the types prescribed by the above procedure.

(4) Are there some possible types of $n$-tuples for which no $n$-tuple exists?

For example, there are, at most, two types of 10-tuples; a 10-tuple for one type has been exhibited but not for the other; one possibility is that none exists for the other type.

6. Numerical results. The writer has computed $\Delta n$ for $n \leq 26$ and determined all the possible types of $n$-tuples for each $n$ in this range; these are tabulated in Table I. It is convenient to describe a given type of $n$-tuple by specifying the smallest prime in the sequence with respect to an appropriate modulus followed by the succession of differences between the constituent primes. It is observed that a kind of "duality" exists between the different types of $n$-tuples for a given $n$ such that if the set of differences for a given $n$-tuple is known, its dual will have these same differences in reverse order. Some $n$-tuples are self dual and do not yield distinct types, such as $n=6$. An examination of Table I shows that for $n=2,4$, and 6 there is only one type of $n$-tuple; for $n=8$, there are 3 types; for $n=3,9,15$, and 22 there are 4 types; for $n=13$ there are 6 types; for all others in the table two types are given, each the dual of the other. In a recent attempt to discover another $n$-tuple that is self dual the writer ascertained that for $n=41$ there are 8 distinct types of possible 41 -tuples.

The numerical results obtained in this investigation were performed on an IBM 701 electronic calculator. Four separate programs were written. Program I was designed to search for 4-tuples; an exhaustive search of all numbers $\leq 5,073,379$ was made and 5494 -tuples were discovered.

Program II was designed to search for 7-tuples of the types

$$
\begin{aligned}
& \text { (A) } 210 x+11,13,17,19,23,29,31 \\
& \text { (B) } 210 x+179,181,187,191,193,197,199 .
\end{aligned}
$$

Seventeen 7-tuples of type (A) and twenty-four of type (B) were discovered in an exhaustive search of all numbers $\leq 157,131,419$. 
TABLE I

\begin{tabular}{|c|c|c|}
\hline$n$ & $\Delta n$ & $m$ \\
\hline 2 & 2 & , \\
\hline 3 & 6 & 30 \\
\hline 3 & 6 & 30 \\
\hline 3 & 6 & 30 \\
\hline 3 & 6 & 30 \\
\hline 4 & 8 & 30 \\
\hline 5 & 12 & 30 \\
\hline 5 & 12 & 30 \\
\hline 6 & 16 & 210 \\
\hline 7 & 20 & 210 \\
\hline 7 & 20 & 210 \\
\hline 8 & 26 & 30 \\
\hline 8 & 26 & 210 \\
\hline 8 & 26 & 210 \\
\hline 9 & 30 & 30 \\
\hline 9 & 30 & 30 \\
\hline 9 & 30 & 210 \\
\hline 9 & 30 & 210 \\
\hline 10 & 32 & 210 \\
\hline 10 & 32 & 210 \\
\hline 11 & 36 & 2310 \\
\hline 11 & 36 & 2310 \\
\hline 12 & 42 & 2310 \\
\hline 12 & 42 & 2310 \\
\hline 13 & 48 & 2310 \\
\hline 13 & 48 & 2310 \\
\hline 13 & 48 & 2310 \\
\hline 13 & 48 & 2310 \\
\hline 13 & 48 & 2730 \\
\hline 13 & 48 & 2730 \\
\hline 14 & 50 & 30030 \\
\hline 14 & 50 & 30030 \\
\hline 15 & 56 & 30030 \\
\hline 15 & 56 & 30030 \\
\hline 15 & 56 & 210 \\
\hline 15 & 56 & 210 \\
\hline 16 & 60 & 30030 \\
\hline 16 & 60 & 30030 \\
\hline 17 & 66 & 30030 \\
\hline 17 & 66 & 30030 \\
\hline 18 & 70 & 30030 \\
\hline 18 & 70 & 30030 \\
\hline & 76 & 10510 \\
\hline & 76 & 10510 \\
\hline & 80 & 30030 \\
\hline 20 & 80 & 30030 \\
\hline & 84 & 30030 \\
\hline & 84 & 30030 \\
\hline & 90 & 10510 \\
\hline & 90 & 10510 \\
\hline & 90 & 10510 \\
\hline & 90 & 10510 \\
\hline & 94 & 10510 \\
\hline & 94 & 10510 \\
\hline & 100 & 10510 \\
\hline & 100 & 10510 \\
\hline & 110 & 30030 \\
\hline & 110 & 30030 \\
\hline & 114 & 99690 \\
\hline & 114 & 99690 \\
\hline
\end{tabular}

$p(\bmod m)$ differences

52

$11 \quad 2,4$

$17 \quad 2,4$

$\begin{array}{lll}7 & 4,2\end{array}$

134,2

$112,4,2$

$112,4,2,4$

$7 \quad 4,2,4,2$

$974,2,4,2,4$

$112,4,2,4,6,2$

$1792,6,4,2,4,2$

$172,4,6,2,6,4,2$

$112,4,2,4,6,2,6$

$1736,2,6,4,2,4,2$

$134,2,4,6,2,6,4,2$

$172,4,6,2,6,4,2,4$

$112,4,2,4,6,2,6,4$

$1694,6,2,6,4,2,4,2$

$112,4,2,4,6,2,6,4,2$

$1672,4,6,2,6,4,2,4,2$

$12712,4,2,4,6,2,6,4,2,4$

$10034,2,4,6,2,6,4,2,4,2$

$12712,4,2,4,6,2,6,4,2,4,6$

$9976,4,2,4,6,2,6,4,2,4,2$

$12712,4,2,4,6,2,6,4,2,4,6,6$

$9916,6,4,2,4,6,2,6,4,2,4,2$

$12592,10,2,4,2,4,6,2,6,4,2,4$

$10034,2,4,6,2,6,4,2,4,2,10,2$

$11992,6,6,4,2,4,6,2,6,4,2,4$

$14834,2,4,6,2,6,4,2,4,6,6,2$

$151312,4,2,4,6,2,6,4,2,4,6,6,2$

$148492,6,6,4,2,4,6,2,6,4,2,4,2$

$151312,4,2,4,6,2,6,4,2,4,6,6,2,6$

$148436,2,6,6,4,2,4,6,2,6,4,2,4,2$

$172,4,6,2,6,4,2,4,6,6,2,6,4,2$

$1372,4,6,2,6,6,4,2,4,6,2,6,4,2$

$69434,2,4,6,2,6,4,2,4,6,6,2,6,4,2$

$230272,4,6,2,6,6,4,2,4,6,2,6,4,2,4$

$69434,2,4,6,2,6,4,2,4,6,6,2,6,4,2,6$

$230216,2,4,6,2,6,6,4,2,4,6,2,6,4,2,4$

$69434,2,4,6,2,6,4,2,4,6,6,2,6,4,2,6,4$

$23017 \quad 4,6,2,4,6,2,6,6,4,2,4,6,2,6,4,2,4$

$2171534,2,4,6,2,6,4,2,4,6,6,2,6,4,2,6,4,6$

$2932816,4,6,2,4,6,2,6,6,4,2,4,6,2,6,4,2,4$

$292,6,4,2,4,6,6,2,6,4,2,6,4,6,8,4,2,4,2$

$299212,4,2,4,8,6,4,6,2,4,6,2,6,6,4,2,4,6,2$

$292,6,4,2,4,6,6,2,6,4,2,6,4,6,8,4,2,4,2,4$

$29917 \quad 4,2,4,2,4,8,6,4,6,2,4,6,2,6,6,4,2,4,6,2$

$194,6,2,6,4,2,4,6,6,2,6,4,2,6,4,6,8,4,2,4,2$

$5104012,4,2,4,8,6,4,6,2,4,6,2,6,6,4,2,4,6,2,6,4$

$236,2,6,4,2,4,6,6,2,6,4,2,6,4,6,8,4,2,4,2,4$

$510397 \quad 4,2,4,2,4,8,6,4,6,2,4,6,2,6,6,4,2,4,6,2,6$

$194,6,2,6,4,2,4,6,6,2,6,4,2,6,4,6,8,4,2,4,2,4$

$510397 \quad 4,2,4,2,4,8,6,4,6,2,4,6,2,6,6,4,2,4,6,2,6,4$

$2171534,2,4,6,2,6,4,2,4,6,6,2,6,4,2,6,4,6,8,4,6,2,4$

$2932574,2,6,4,8,6,4,6,2,4,6,2,6,6,4,2,4,6,2,6,4,2,4$

$292,6,4,2,4,6,6,2,6,4,2,6,4,6,8,4,2,4,2,4,14,4,6,2$

$298912,6,4,14,4,2,4,2,4,8,6,4,6,2,4,6,2,6,6,4,2,4,6,2$

$34649992,12,4,2,4,6,2,6,4,2,4,6,8,6,4,2,6,4,6,8,4,2,4,2,4$

$62345774,2,4,2,4,8,6,4,6,2,4,6,8,6,4,2,4,6,2,6,4,2,4,12,2$ 
TABLE II

1 st term of $n$-tuple $\quad n$

Type

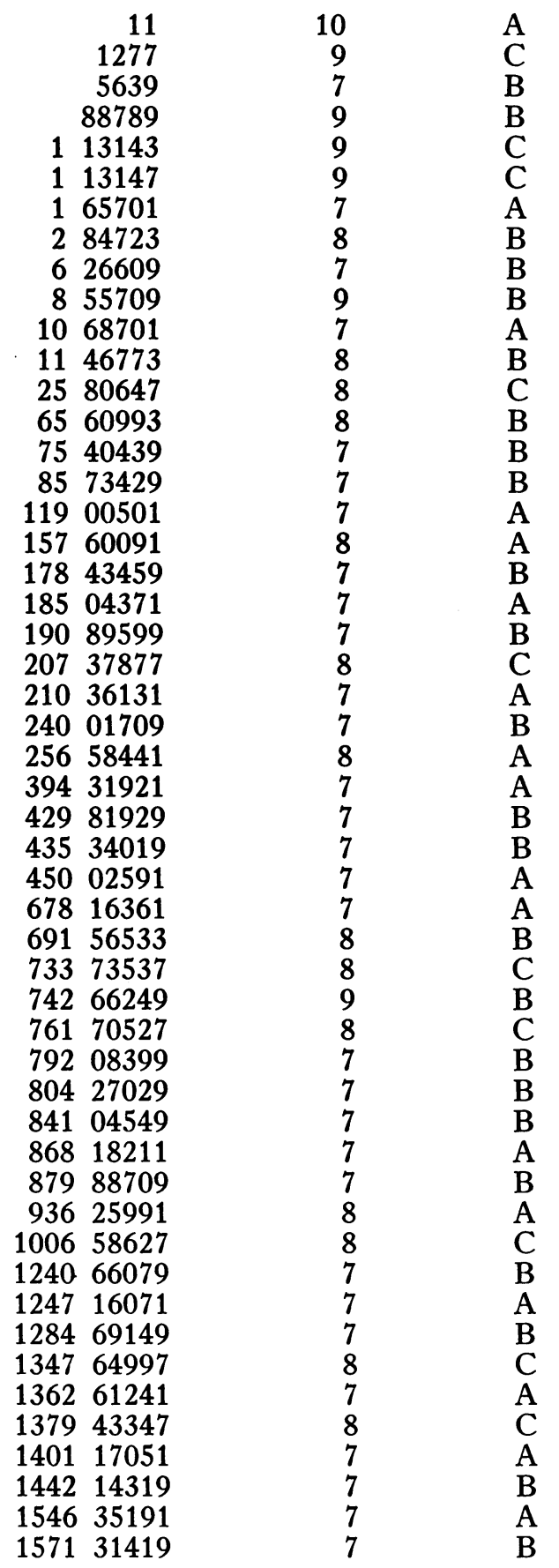


Program III was designed to search for 8-tuples of the type

(C) $30 x+17,19,23,29,31,37,41,43$.

Ten 8-tuples of this type were obtained in a search of all numbers $\leq 137,943,347$.

Program IV was the unsuccessful attempt to find a 19-tuple. As a check on the above calculations program II was recently re-run on an IBM 650 magnetic drum calculator; there was 100 percent agreement between the results of the two machines.

An examination of Table I shows that all $n$-tuples in a given range of numbers for $7 \leq n \leq 15$ can be determined by examining only the $n$-tuples obtained from the three types given above. This subsequent calculation yielded three 8-tuples and one 10-tuple for type (A), four 8-tuples and three 9-tuples for type (B) and three 9-tuples for type (C). These results are tabulated in Table II. Since a 9tuple may also be an 8-tuple, and a 7-tuple, etc., the highest ranking designation is given in each case.

7. Conclusion. The prime pair problem has been shown to be the simplest case of a more general problem. Essentially, the problem poses two questions:

(5) How "clustered" can a given number of primes be?

(6) How many such "clusters" are there?

The first question is considered equivalent to the question, what is the smallest range of natural numbers that can contain $n$ primes? The scheme proposed in this paper gives a necessary range; it is suggested but not proved generally that this range is sufficient. It is proved to be both necessary and sufficient for all $n$-tuples for $n \leq 10$.

G. H. Hardy [3] and W. W. Ball [4] have made conjectures concerning the number of prime pairs and higher $n$-tuples under a given magnitude but the results of this investigation are too meager to expect any degree of accuracy from the formulas that have been proposed.

The programs for this investigation were not the most efficient since the writer had a limited amount of time to write, debug and run them. With a more elegant approach to the programming and by using the more sophisticated logic of the IBM 704 these calculations could be accelerated by a factor of 200 or more.

The writer wishes to express his thanks to the referee for his comments and suggestions.

Herschel F. SMith

Applied Science Division

International Business Machines

Chicago, Illinois

1. A. De Polignac, Nouvelles Annales de Math., v. 8, 1849, p. 428.

2. Edmund Landau, Vorlesungen über Zahlentheorie, Chelsea Pub. Co., New York, v. 1, 1947, p. 71.

3. G. H. Hardy \& J. E. Litrlewood, "Some problems of 'Partitio Numerorum'; III: On the expression of a number as a sum of primes," Acta Math., v. 44, 1923, p. 1-70.

4. W. W. BALL, Mathematical Recreations and Essays, 11th ed., p. 64. 